\title{
Study of Infectious Diarrheas in Transylvania-Romania
}

\section{Liana Monica Deac*}

National Public Health Institute, Public Health Center, Cluj-Napoca, Romania.

Correspondence to: Liana Monica Deac, National Public Health Institute, Public Health Center, Cluj- Napoca, Romania.

Received date: January 30, 2021; Accepted date: February 12, 2021; Published date: February 20, 2021

Citation: Deac LM (2021) Study of Infectious Diarrheas in Transylvania-Romania. J Med Res Surg 2(1): pp. 1-3.

Copyright: (C2021 Deac LM. This is an open-access article distributed under the terms of the Creative Commons Attribution License, which permits unrestricted use, distribution and reproduction in any medium, provided the original author and source are credited.

\section{ABSTRACT}

"Diarrhea" is an alteration in a normal bowel movement characterized by an increase in the water content, volume or frequency of stools. Acute diarrhea of infectious etiology often referred to as gastroenteritis and is typically associated with clinical signs and symptoms including nausea, vomiting, abdominal pain and cramps, bloating, flatulence, fever, the passage of bloody stools, tenesmus, and fecal urgency. Infectious diarrheal diseases are the second leading cause of morbidity and mortality worldwide. It is diarrhea we have also found out during a large 3 years study 2017-2020 in TransylvaniaRomania. 3577 number of cases were transmitted during this time by the 12 territory sanitary policies to the Epidemiology Department from the Public Health Center Cluj. Those were mostly diagnosed by the territorial family doctors in the region and more than $20 \%$, needed hospitalization for several days because of the mentioned disease disorders 3 children died during this time because of severe complications. The detected infectious microbial etiology were determined in authorized laboratories in which it was identified as Shigella spp., Salmonella spp., Campylobacter spp. and Yersinia spp. or Rotavirus Giardia and Fungi species. Most numbers of cases appeared in children, followed by elderly or adult people, as quantified number of determination.

In light of these data, acute diarrheal illness had to be considered a major public health issue against which control efforts are needed. Public health surveillance and response in the field of infectious acute diarrhea include obligatory strategies of infection control.

\section{Keywords:}

Diarrhea symptoms, Etiology, Health survey

\section{Introduction}

According to the World Health Organization (WHO) and UNICEF, there are about 2 billion cases of diarrheal disease worldwide every year and 1.9 million children younger than 5 years of age perish from diarrhea each year mostly in developing countries. In industrialized countries, relatively few patients die from diarrhea but the disease continues to be an important cause of morbidity that is associated with substantial health care costs. However, the morbidity from diarrheal diseases has remained relatively constant during the past 2 decades [4].

Because of these data, the morbidity caused by infectious diarrhea which appeared in Transylvania region-Romania was studied in the Epidemiology Department of the Public Health Center for 3 years during 2017-2020. Despite the economic and societal burdens of diarrheal illnesses, few clinical guidelines exist worldwide for the diagnosis and treatment of persons with suspected infectious diarrhea. For that considerable clinical practice has to be observed and demonstrate a clear need for a clinical diagnostic improved guideline.

\section{Materials and Methods}

By using an analytical descriptive epidemiological study, 3577 cases were analyzed of infectious diarrhea in the population to figure out all representative aspects of this pathology in children, adults, and elderly people. Data recruitment were ordered by the Ro national public health guidelines and was transmitted yearly by the territory sanitary police units. For every 3 years from May to November and sent to the Public Health Center Cluj-Epidemiology Department where the epidemiologic study was done and received all data interpretations.

\section{Results and Comments}

Total 3577 cases of Infectious diarrhea was present in the Transylvania region- Ro, the relevant data for a yearly comment is shown in Figure 1.

During the study most of the diseases were present in the first year i.e. $37 \%$, followed by $35.5 \%$ in the second year and $29.5 \%$ in the third one. In all 3 years, most of this pathology appeared in the summer season during July and August. In our entire territory studied there was not a significant estimate difference between the yearly done activities for it. The case incidence for the disease arrived at $65 \%$ in the seasonal morbidity and was specially represented during the mentioned summer months July and August.

The Significant figured out was even, that infectious diarrhea existed at each age decade, but more significant in children with 63\%, as shown in Figure 2.

The disease was present in children from 0-14 age representative and in the decade the most reported cases were of children from 1-4 age. In adults, infectious diarrhea was remarked as 


\section{Yearly cases reported during : 2017-2020}

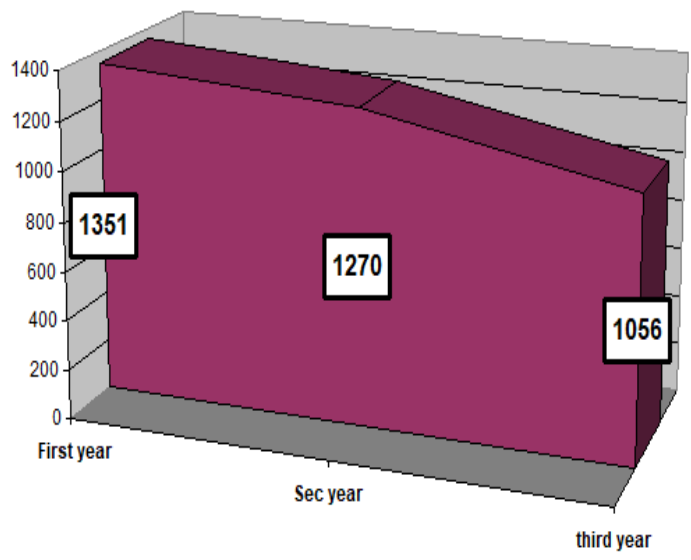

Figure 1: Infectious diarrhea during 2017-2020.

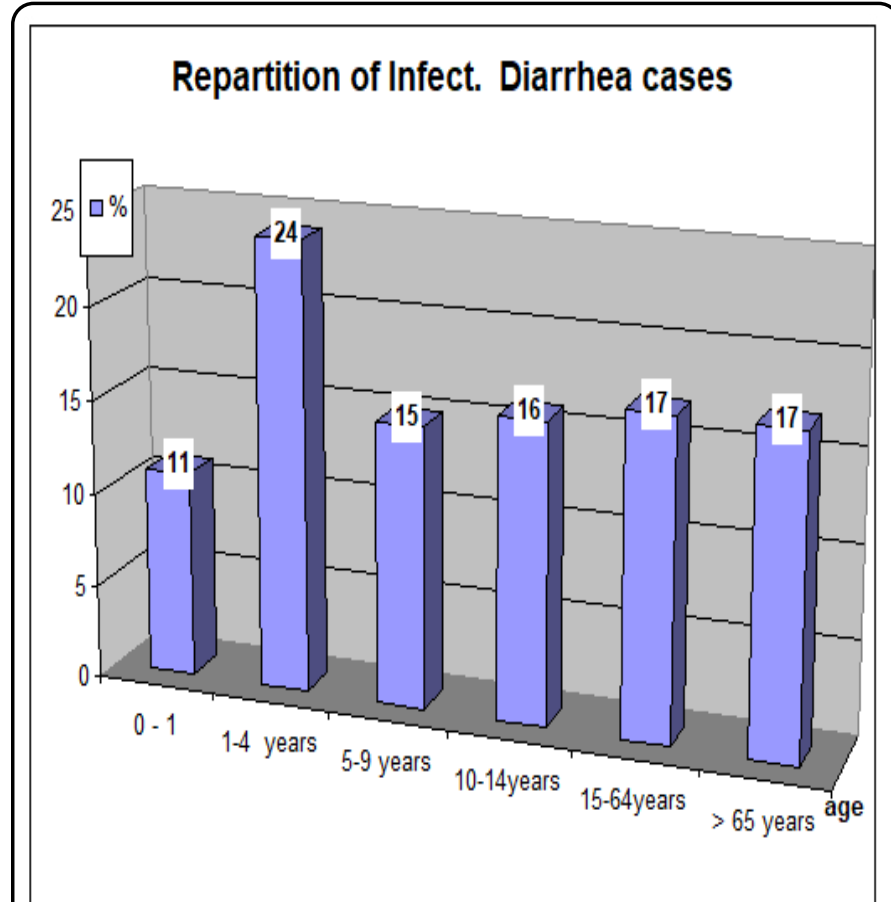

Figure 2: Distribution of the disease.

$17 \%$, as well in elderly people this pathology was represented in the same number. $27 \%$ of cases from children and adults needed short hospitalization as they were accused of dehydration. During the entire mentioned period of the observation, a specific adequate fluid and electrolyte replacement was used as a key treatment to manage diarrheal illnesses with full maintenance. Even though after this specific therapy 3 children under 5 years of age were died because of severe complications. Organic failure was estimated to be the cause of their deaths.

In the developing world, there is also a growing awareness of the potentially huge impact of long-term disability caused by repeated early childhood enteric infections. It is estimated that there are 3.1 million worldwide deaths due to diarrhea per year (18400 per day), mostly of young children in developing areas [1]. Globally, due to diarrhea annual deaths occur mainly in young children and the number of deaths is 1000 folds higher than in the United States where most of those who die of diarrheal illness are elderly [2].

The severity and type of illness can be defined through clinical and epidemiological evaluation. For clinicians, early diagnosis of an acute episode of diarrhea can lead to interventions that alleviate symptoms and prevent secondary possible transmission. For public health practitioners, prompt notification of pathogen-specific diagnosis and bacterial isolates through public health surveillance can lower rates of transmission and lead to timely detection and control of outbreaks. Because both clinicians and public health practitioners share overlapping responsibilities for the diagnosis, management, and prevention of infectious diarrheal diseases must contain recommendations and specific guidelines for both groups [5].

Diarrheal illness is a worldwide problem with substantial regional variation in the prevalence of specific pathogens. Diagnostic testing of stool specimens was indicated for patients up to an existing national guideline to identify them. For that, it was significant to be figured out the etiological aspects concerning all detected infectious diarrhea of all the dates which came from the several territory laboratories as investigation. All these data were identified to present the microbiological discovered aspects in the several presented situations during the 3 years study. With the help of this, it was possible to make an overview concerning the heterogenic microorganisms that made the appearance of the infectious diarrhea diseases in the Transylvania population. The incidence of this aspect is presented in Figure 3.

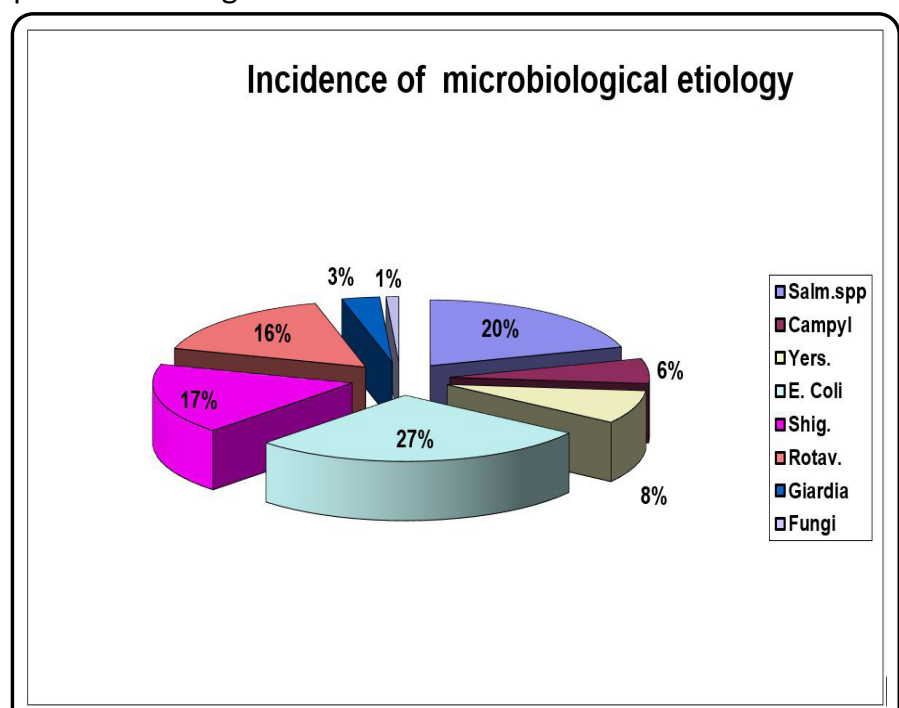

Figure 3: Overview of the detected etiological aspects.

Most of the species belonged to the Enterobacteriaceae Family, from that E. coli was $27 \%$, Salmonella spp. was $20 \%$; Shigella spp. was $17 \%$. Important was to figure out the invasive E. coli in their stool samples that caused the death of the 3 children who were under 5 age. It was also important to be remarked that the presence of Yersinia spp. was $8 \%$ and of Campylobacter spp. was $6 \%$ in other patients' samples. The presence of these different bacterial aspects, were for $E$. Coli in the disease cases, at almost in children faces identified but all other mentioned species were even in adults, or elderly people samples detected by the territory laboratories. 
The observation of this study also mentioned that $17 \%$ of the Rotavirus was also present which mostly were present in the infectious diarrhea of children as an etiological aspect. $3 \%$ of children have had even Giardia and $1 \%$ of elderly people have had Fungi in their preserved tools. Many of these organisms are easily transmitted through food or water or from one person to another as the epidemiological study figured out results in the territory.

There are various interpretations of what is considered medically indicated for evaluating persons with diarrhea. Stool cultures are often viewed as tests with high importance [3]. With the rapid globalization and industrialization of our food supply and with a multiplicity of recognized pathogens and diagnostic tools, the challenges of determining optimal, cost-effective means for appropriate diagnosis, clinical management, and public health control of diarrheal illnesses are great [6]. This will continue to evolve as improved understanding of pathogenesis and development and use of inexpensive, rapid tests improve diagnosis and management of infectious diarrheal illness, one of the most common clinical syndromes in our society. The lack of a specific diagnosis can hinder the appropriate management and treatment of many infections. Observation of patients is recommendations intended to provide clinicians and public health practitioners with a consensus-based document that will aid in the management of acute diarrhea by addressing which patients to test, what tests to order, next what medical treatments to use and what steps to take to ensure that appropriate public health actions are implemented.

\section{Conclusion}

There was no specific difference of data in the 3 studied years and infectious diarrhea appeared mostly during the summer months.

Children were more involved in the infectious diarrhea incidence, but each patient should be observed about potential epidemiological risk factors, for any diarrheal diseases or for their possible spread in the territory.

Infection causes as bacterial, viral and parasitic were present among all cases, as the guidelines for the pathology were recommended to be detected in the done study.

All diarrheal pathogens had to be identified. Evaluating persons with the disease and identifying each enteric infection that requires specific control measures in clinics and health care activity groups.

To reduce the morbidity and mortality associated with infectious diarrhea needs clinical and public health studies must be closely done to identify optimal diagnostic, treatment and prevention methods.

\section{References}

1. Adkins HJ, Escamilla J, Santiago LT, et al. (1987) Two-year survey of etiologic agents of diarrheal disease at San Lazaro Hospital, Manila, Republic of the Philippines. J Clin Microbiol 25(7): pp. 1143-1147.

2. Riley MR, Bass D (2008) Pediatric Gastroenterology, https://www.sciencedirect.com/book/9780323032803/ pediatric-gastroenterology

3. Guerrant RL, Gilder TV, Steiner TS, et al. (2001) Practice guidelines for the management of infectious diarrhea. Clin Infect Dis 32(3): pp. 331-351.

4. Snyder JD, Merson MH (1982) The magnitude of the global problem of acute diarrheal disease: a review of active surveillance data. Bull World Health Organ 60(4):pp. 605-613.

5. WHO Program for the Control of Diarrhoeal Diseases (1989) $\mathrm{CD}$ update on persistent diarrhea.

6. WHO (1993) The management and prevention of acute diarrhea: practical guidelines. 3rd ed. Geneva: World Health Organization. 